\title{
Free water in fuel sensor using fiber long period grating
}

\author{
W. Zhang ${ }^{*}$, S. Grice, K. Sugden, I. Bennion \\ Photonics Research Group, Aston University, Birmingham, B4 7ET, UK,
}

\begin{abstract}
A fiber optic free water in fuel (WIF) sensor is proposed by utilizing a long period fiber grating (LPFG). The existence of free water in fuel is indicated by the appearance of a characteristic loss band. The free water level in fuel can be determined by measuring the transmissions of two characteristic loss bands.
\end{abstract}

Keywords: Long period fiber grating, free water in fuel, surrounding refractive index, characteristic loss band

\section{INTRODUCTION}

In aircraft fuel tanks the fuel is subjected to pressure and temperature changes and condensation may add to the water content of the fuel. Undissolved (free) water in aviation jet fuel is also considered to be a contaminant, because if left uncontrolled it can lead to the plugging of filters, when the water is frozen, or microbiological growth in places where the water settles. In extreme cases, it could also cause power stability problems in an engine if a large amount passes through in place of fuel. Free water usually settles at the lowest point in the tank. Once the water has settled, good housekeeping practices will ensure that it is removed by draining at regular intervals. Therefore a reliable WIF sensor is required to monitor free water level in fuel. Conventionally the WIF sensor uses the difference of electric conductivity through water and fuel by 2 electrodes. However optical sensors are more desirable in these areas because of the hazards posed by sparks in potentially explosive environments. There has no optical free water in fuel sensor reported. In this work we propose the first optical free water in fuel sensor based on a long period fiber grating.

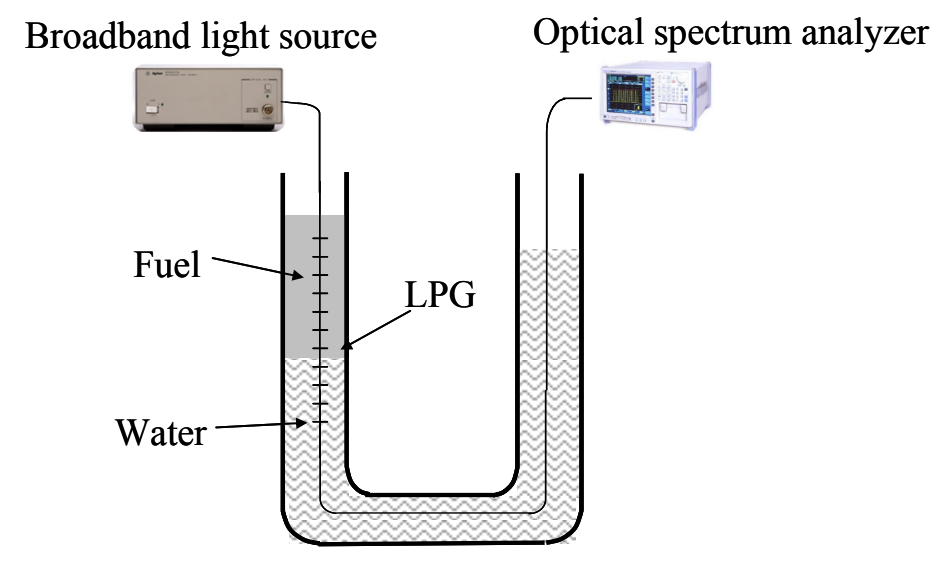

Fig. 1: Experiment setup

*w.zhang@aston.ac.uk

21st International Conference on Optical Fiber Sensors, edited by Wojtek J. Bock, Jacques Albert, Xiaoyi Bao, Proc. of SPIE Vol. 7753, 77537C · C 2011 SPIE · CCC code: 0277-786X/11/\$18 · doi: 10.1117/12.885913 


\section{EXPERIMENT AND RESULT}

For optical sensing applications, LPFGs have been implemented as temperature, strain, refractive-index sensors as well as sensing demodulators ${ }^{[1]}$. The fact that light coupling involves cladding modes means that the LPFG spectral response is strongly influenced by the optical properties of the cladding and the surrounding medium ${ }^{[2]}$. This unique feature has yielded several novel sensing concepts and devices. Its sensitivity to external refractive index has been exploited for chemical sensing applications.

For the investigations performed, the LPFG used was fabricated by exposing SMF-28 fibre to UV irradiation. The LPFG was $100 \mathrm{~mm}$ in length and had a period of $500 \mu \mathrm{m}$. The basic configuration for the free water in fuel sensor is depicted in Fig. 1. The LPFG was first exposed to unleaded fuel with the refractive index of $\sim 1.42$ and the spectrum of the LPFG was monitored using a broadband source and an optical spectrum analyzer with a resolution of $0.1 \mathrm{~nm}$. In this work the LPFG was suspended between two fixed points in the left side of a $U$ type glass tube. Water was added to the U-tube from the right side to push the petrol level up thus increase the water level in fuel.

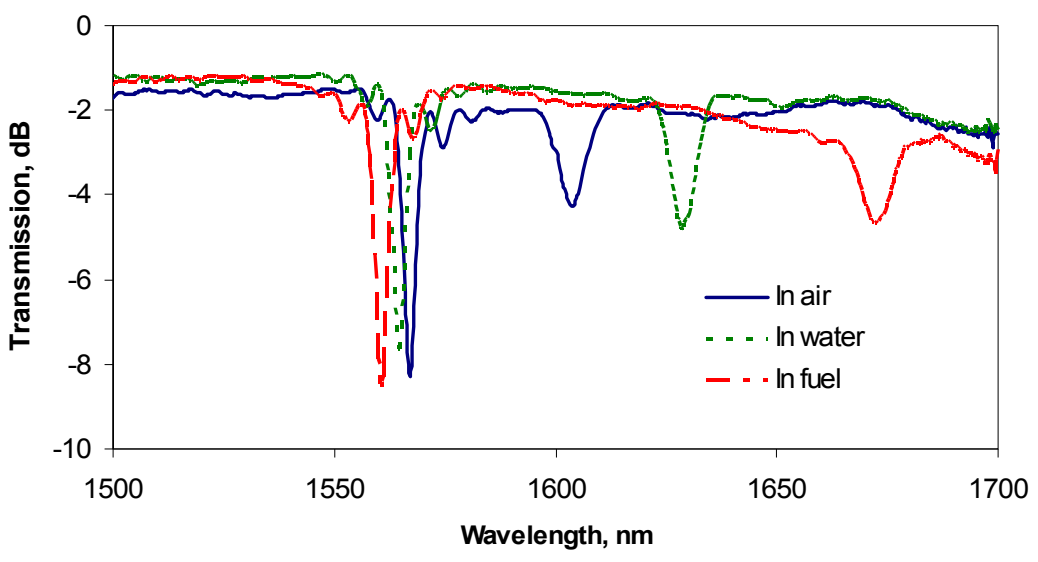

Fig. 2 Spectral responses of LPFG in different surrounding media

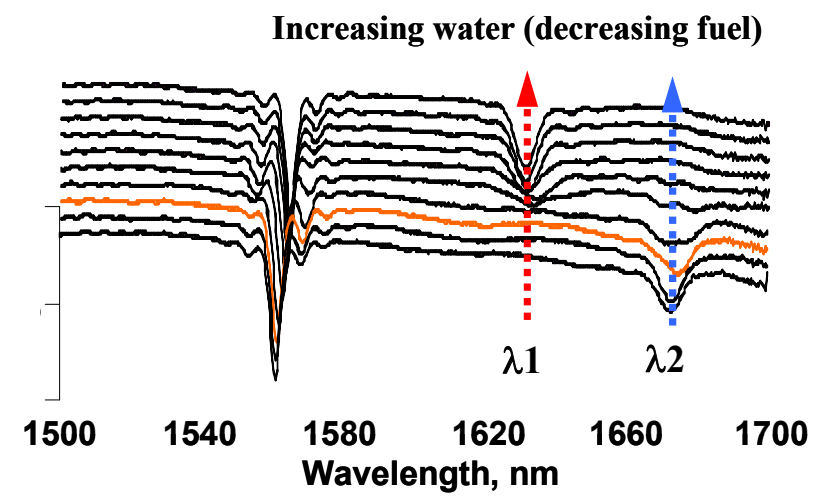

Fig. 3 LPFG spectral responses against free water level

Figure 2 shows the measured transmission spectra of the LPFG when it was fully immersed in air, water and fuel (unleaded petrol), respectively. In this spectral range two loss bands exist. When the LPG placed in air, water and unleaded petrol, the surrounding refractive index varied and the corresponding two cladding modes clearly show the opposite chromatic dispersion change. 
The loss band at the longer wavelength showed a more interesting feature when we started looking into the free water level in fuel. The changes of the resonant wavelengths against free water level change in fuel was recorded and is depicted in Fig. 3, from which one can clearly see the resonant wavelength of the first loss band shows a small red shift against the increased free water level. The resonant wavelength of the second loss band was at $\sim 1674 \mathrm{~nm}$ when it was fully immersed in petrol. As the free water level increased, this resonant wavelength showed a blue shift and the peak strength decreased. The resonance disappeared when the free water reached a certain level. Meanwhile a new loss band gradually appeared at a shorter wavelength (around $1630 \mathrm{~nm}$ ) and its peak strength increased with the increased free water level. It should be pointed out that this new loss band at $1630 \mathrm{~nm}$ is characteristically related to water. Once this loss band appears it indicates the existence of free water in fuel.

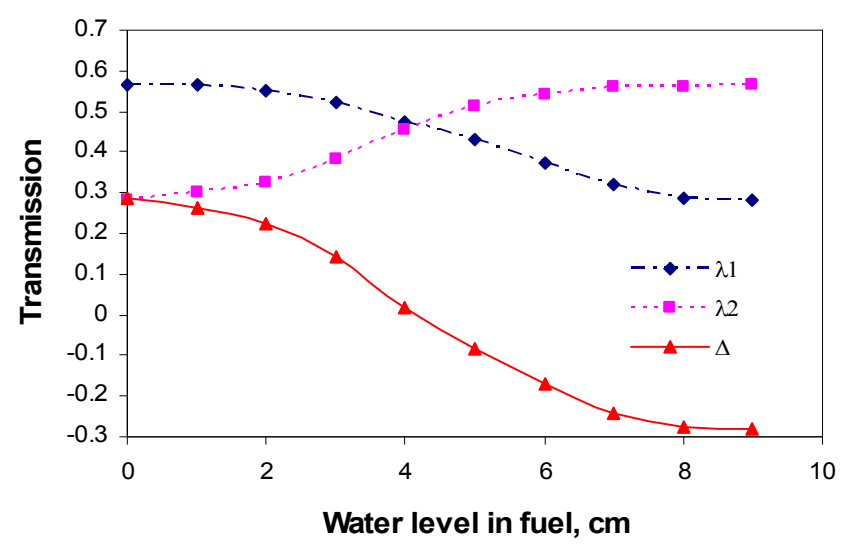

Fig. 4 LPFG transmission at specific wavelengths vs. increased free water level in fuel

From Fig. 3 it can be clearly seen that the free water level in the petrol is related to the strength of the loss band at either $\lambda 1$ or $\lambda 2$. These two loss bands show an opposite strength change against varying free water level. By measuring the transmission responses at these two wavelengths the free water level in the fuel can be determined. The transmission response of LPFG sensor at these two specific wavelengths was measured and plotted in Fig. 4. From this figure one can see that the sensor response shows a monotonic decrease and increase at $\lambda 1$ and $\lambda 2$ against the increased free water level, respectively. The difference of these two values is plotted in the figure as well, which gives larger responsivity against the corresponding free water level.

\section{DISCUSSION AND CONCLUSION}

In this work the initial surrounding medium of the LPFG is fuel (unleaded petrol) with the refractive index of $\sim 1.42$. If the LPFG is partly immersed in the free water then two LPFGs are formed in series. The second (new) one has the length equal to the immersed length and the surrounding RI equal to that of the free water. From the figures 2 and 3 one can conclude that the loss band at $\lambda 1(\sim 1630 \mathrm{~nm})$ characterizes the phase match condition for the surrounding medium of water, and the loss band at $\lambda 2(\sim 1674 \mathrm{~nm})$ for the surrounding medium of fuel. As a result the loss band at $\lambda 1$ representing the new LPFG becomes stronger with the increased free water level and the loss band at $\lambda 2$ representing the original LPFG shows the opposite tendency.

Clearly the responsivity of this LPFG sensor is lower when the free water level reaches the two ends of the LPFG. This may affect the performance for monitoring small free water level. The sensitivity can potentially be improved by either designing LPFG with higher response or simply placing the LPFG in tilted position so to increase the length of the LPFG interacting with the free water.

We have successfully demonstrated a fiber optic free water in fuel sensor by using a long period fiber grating. The 
existence of free water in fuel can be indicated by the appearance of the loss band at characteristic wavelength $\lambda 1$ without ambiguity. The free water level in fuel can be determined by measuring the transmission responses of two characteristic loss bands.

\section{ACKNOWLEDGEMENT}

The work was sponsored under the UK TSB project "Integrated Wing."

\section{REFERENCES}

[1] Shu, X., Zhang, L., Bennion, I., "Sensitivity characteristics of long-period fiber gratings" J. Lightwave Technol. 20(2), 255-266 (2002).

[2] Patrick, H.J. Kersey, A.D. Bucholtz, F., "Analysis of the response of long period fiber gratings to external index of refraction,” J. Lightwave Technol., 16(9), 1606-1612 (1998). 